\title{
Toughening Effect of Continuous Fiber Bundles
}

\author{
Xinguo Kong AND Yu QIAO* \\ Department of Civil Engineering \\ University of Akron \\ Akron, $\mathrm{OH}$ 44325-3905, USA
}

(Received May 6, 2004)

(Accepted November 8, 2004)

\begin{abstract}
In a continuous fiber reinforced brittle matrix composite, the transverse fracture is often dominated by the crack trapping and bridging effects as well as the fiber breakage. In this article, the toughening effect of flat fiber bundles with the cross-sectional aspect ratio, $\rho$, ranging from 0.05 to 5 is discussed in the context of energy analysis. With a constant size/spacing ratio of the fiber bundles, the fracture resistance increases monotonically with $\rho$, primarily due to the influences on the crack front behavior. The elastic properties of matrix and the crack length have little effect on the critical energy release rate.
\end{abstract}

KEY WORDS: fiber reinforced composites, fracture resistance, aspect ratio.

\section{INTRODUCTION}

$\mathbf{T}$ HE CRACK PROPAGATION in fiber reinforced composites (FRC) is of prime technological importance. In engineering practice, very often the reliable service performance of FRC is required even in the post critical stage. The catastrophic crack advance that rapidly leads to the final failure is unacceptable under any condition $[1,2]$. Along the fiber direction, the resistance to crack advance is mostly determined by the fiber-matrix bonding, and for multilayer structures the interlaminar fracture can be dominant [3-5]. Along the transverse direction, however, the fibers are usually impenetrable and act as obstacles to the crack propagation. The crack front is forced to advance in a nonuniform manner across fiber arrays, and the crack flanks can be bridged together as the front moves forward [6]. Eventually the fibers are broken apart, which further increases the fracture work. This phenomenon is specifically important when fibermatrix separation is energetically unfavorable under certain loading modes or in multiaxial composites. Under this condition, the crack growth direction changes frequently and the fracture path can be quite tortuous. The crack front penetrates into

*Author to whom correspondence should be addressed. E-mail: yqiao@uakron.edu

Journal of COMPOSITE MATERIALS, Vol. 39, No. 17/2005 
the 'weak' area of the composite, followed by the dispersed out-of-plane failure. Over many years, certain empirical design strategies have evolved based on experiments under either quasi-static or dynamic loadings $[7,8]$. In addition, a large number of numerical simulations have explored the structure parameters, such as the fiber strength and the fiber volume fraction [9-12].

In the processing development of FRC, reinforcing the matrix with the flat fiber bundles has been an active research area for decades, primarily due to the superior mechanical properties and the excellent cost-performance balance. With the optimized structure, the stiffness and strength are enhanced considerably [2], and other properties, such as thermal conductivity and electrical resistivity can be adjusted conveniently in broad ranges $[13,14]$. Usually a fiber bundle consists of many layers either braided or glued together, and a variety of surface treatment techniques have been established such that the fiber-matrix bonding can be stronger than the fiber bundle itself [15].

The aspect ratio of the cross sections of fiber bundles, $\rho$, is a vital factor affecting the FRC performance. It comes in by influencing the stress concentration, the effective reinforcement area, the mode of fracture, etc. In order to optimize the processing techniques, it is essential to understand the crack-fiber interaction. Although substantial attention has been given to the crack tip stress/strain field analysis for highly heterogeneous environments [9-12], currently there is still no satisfactory model that can predict the failure criterion accurately.

In view of the above considerations, in this article we will develop a numerical model so as to relate the fracture resistance offered by the fiber bundles to important design variables through an energy analysis, with the computationally expensive simulation of crack front profile evolution being avoided. The influence of the fiber volume fraction and the cross-sectional size and aspect ratio of the fiber bundles is discussed in considerable detail. Note that in the following discussion, the cross sections of the fiber bundles are assumed elliptical. In actual composites, the relatively sharp corners of the fiber bundles may cause complicated additional stress concentrations. However, as long as the fiber-matrix bonding is perfect, its influence on the energy variation should be secondary.

\section{GOVERNING EQUATIONS}

Consider the fracture across a regular array of fiber bundles perfectly bonded to a brittle matrix, as depicted in Figure 1. The fracture mode is cleavage. When the crack front encounters the fiber bundle array, it bows into the matrix as the stress intensity at the crack tip increases. The cross-sectional aspect ratio of a fiber bundle is defined as $\rho=b / a$, with $a$ and $b$ being the lengths of the semimajor axes along the crack advance direction and the initial crack front, respectively. Due to the barrier effect of the fiber bundles, the front penetration is stable. As the penetration depth increases, eventually the two sections of the crack front on both sides of a fiber bundle merge. Thus, a new front is formed ahead of the fiber bundle array and it moves forward, leaving the fibers that bridge across the crack flanks behind.

In order to calculate the strain energy variation, consider the double-cantilever-beam (DCB) specimen depicted in Figure 2. Initially the crack tip is at a point 'A', where the matrix is toughened by an array of fiber bundles. The stress intensity at the crack tip increases with the crack opening displacement, $\delta_{\mathrm{o}}$. When $\delta_{\mathrm{o}}$ reaches the critical value, 


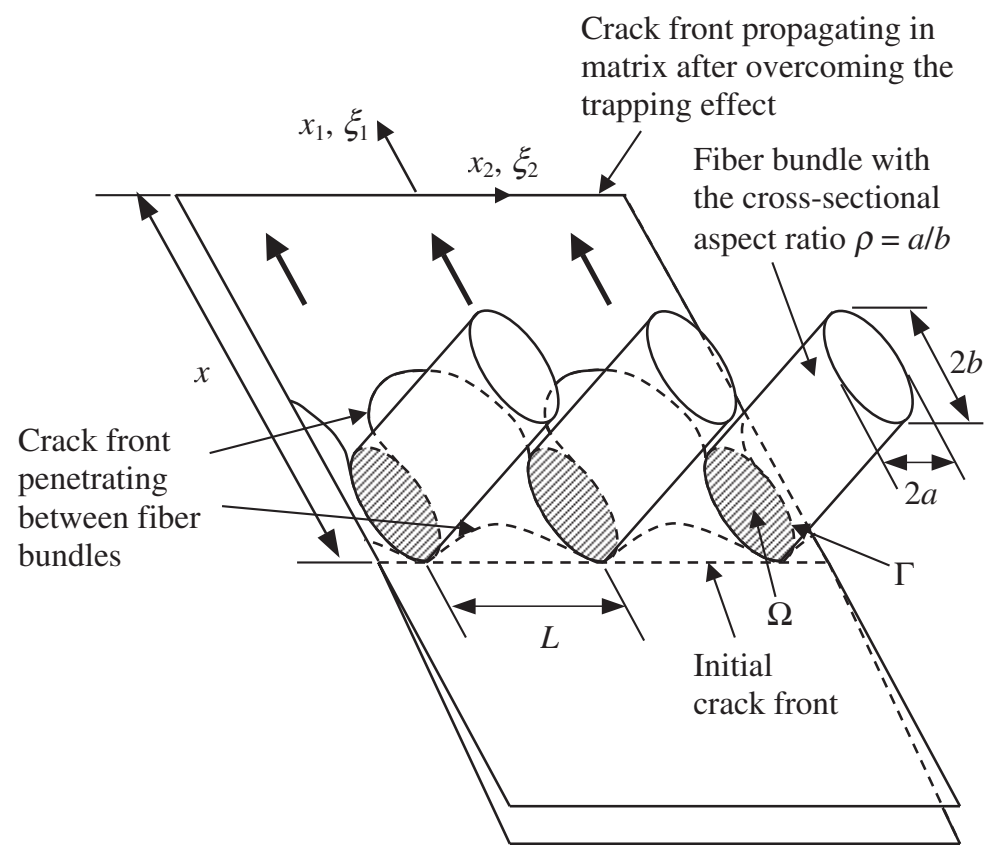

Figure 1. A schematic diagram of the crack front overcoming a regular array of fiber bundles.

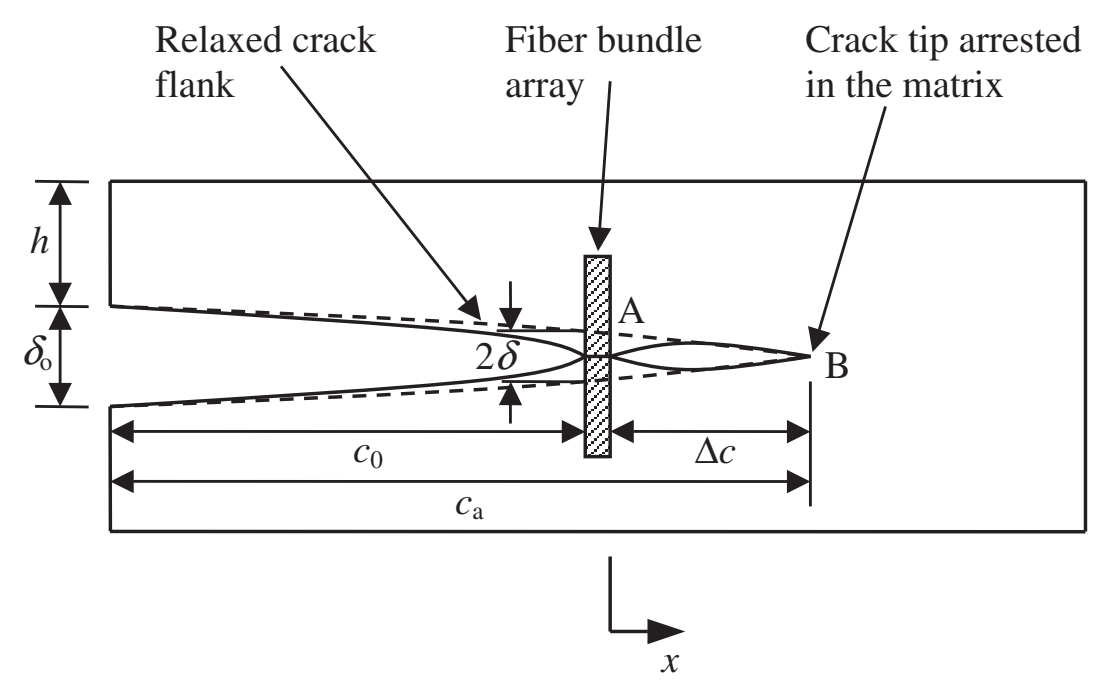

Figure 2. The double-cantilever-beam specimen reinforced by a single array of fiber bundles.

the crack front bypasses the fiber bundle array and, since the fracture resistance offered by the fiber bundle array, $G_{\mathrm{fa}}$, is larger than that of the matrix, $G_{\mathrm{m}}$, the crack will keep growing as $\delta_{\mathrm{o}}$ is kept constant. With the increase of crack length, the stress intensity factor, $K$, decreases, and eventually the crack stops at a point 'B', where, $K$ equals the critical value to arrest the propagating front. If there were a graded ligament ahead of the fiber 
bundle array between the points 'A' and 'B' such that the local fracture resistance is always the same as the energy release rate, the crack growth would be quasi-static and the criterion of crack arrest in the matrix can be stated as:

$$
G=G_{m}
$$

where $G=\left(1-v^{2}\right) K^{2} / E$ is the energy release rate, with $E$ and $v$ being the Young's modulus and the Poisson's ratio, respectively. It is clear that in the actual composite, such a graded ligament does not exist. However, since the behavior of the crack front after the fiber bundle array is bypassed does not affect the penetration process, the value of $G_{\mathrm{fa}}$ calculated for such an 'imaginary' sample is the same as that of the actual FRC. Note that, in the graded sample the calculated distance between the points ' $A$ ' and 'B', i.e., the crack growth length $\Delta c$, is smaller than the actual value.

As will become clear shortly, the geometry of the DCB sample does not affect $G_{\mathrm{fa}}$. Thus, we can choose the range of the height of DCB arm, $h$, and the initial crack length, $c_{0}$, such that the strain energy can be analyzed by the basic beam theory, according to which

$$
U=\frac{E h^{3} \delta_{\mathrm{o}}^{2}}{16 c^{3}}=\frac{c G}{3}
$$

where $U$ is the strain energy per unit thickness, and $c$ is the crack length. Differentiating Equation (2) gives the fracture resistance of the array of fiber bundles

$$
G_{\mathrm{fa}}=\frac{3}{16} \frac{E h^{3} \delta_{\mathrm{o}}^{2}}{c_{0}^{4}} .
$$

When the crack growth distance is $x$, if the fiber bundle array did not exist, the energy release rate is

$$
G_{\mathrm{c}}(x)=\frac{3}{16} \frac{E h^{3} \delta_{\mathrm{o}}^{2}}{\left(c_{0}+x\right)^{4}},
$$

that is,

$$
\hat{G}(x)=\left(\frac{c_{0}}{c_{0}+x}\right)^{4} \quad \text { or } \quad \hat{K}(x)=\left(\frac{c_{0}}{c_{0}+x}\right)^{2},
$$

where $\hat{G}=G_{\mathrm{c}} / G_{\mathrm{fa}}$ and $\hat{K}=K_{\mathrm{c}} / K_{\mathrm{fa}}$, with $K_{\mathrm{c}}=\sqrt{E G_{\mathrm{c}} /\left(1-v^{2}\right)}$ and $K_{\mathrm{fa}}=\sqrt{E G_{\mathrm{fa}} /\left(1-v^{2}\right)}$ being the stress intensity factors. As shown in Figure 2, as the crack advances, the fiber bundles are subjected to bridging forces along the fiber-matrix boundary, $\Gamma$, which result in the local crack closure. With the influence of the bridging forces, the stress intensity factor along the advancing crack front $x$ away from the initial crack tip is [16]

$$
\tilde{K}\left(x_{2}\right)=\hat{K}(x)+\int_{\Gamma} H(s, \vec{\xi}) P(\vec{\xi}) d \vec{\xi}
$$


where $\vec{x}=\left(x_{1}, x_{2}\right)$ is the global coordinate system; $\vec{\xi}=\left(\xi_{1}, \xi_{2}\right)$ is the local coordinate system on a fiber bundle; $x_{1}$ and $\xi_{1}$ are parallel to the crack propagation direction; $x_{2}$ and $\xi_{2}$ are parallel to the crack front; $P(\vec{\xi})$ is the bridging force; and $H(s, \vec{\xi})=\sqrt{2 / \pi^{3}} \sqrt{-\xi_{1} /\left(s^{2}+\xi_{1}^{2}\right)^{2}}$, with $s=\left|x_{2}-\xi_{2}\right|$. The effective energy release rate is

$$
G_{\mathrm{R}}(x)=\frac{\left(1-v^{2}\right) \bar{K}^{2}}{E}
$$

where, $\bar{K}=\int_{0}^{L} \tilde{K} d x_{2}$, and $L$ is the center-to-center distance of the fiber bundles. Note that to keep the crack growth quasi-static, the boundary conditions of $G_{\mathrm{R}}$

$$
G_{\mathrm{R}}(0)=G_{\mathrm{fa}}
$$

and

$$
G_{R}(\Delta c)=G_{m}
$$

must be satisfied.

The bridging force can be calculated by solving the integral equation [12]

$$
2 \hat{K}(x) \sqrt{\frac{x_{1}}{2 \pi}}+\int_{\Gamma} \hat{H}(\vec{x}, \vec{\xi}) P(\vec{\xi}) d \vec{\xi}=0 \quad\left(\text { for } \vec{x} \in \Gamma^{\prime}\right)
$$

where $\hat{H}(\vec{x}, \vec{\xi})=\arctan \left\{2 \sqrt{x_{1} \xi_{1}} /\left[\left(x_{1}-\xi_{1}\right)^{2}+\left(x_{2}-\xi_{2}\right)^{2}\right]\right\}$ and $\Gamma^{\prime}$ denotes the boundary of a single fiber bundle. The first term in the left-hand side is proportional to the relaxed crack opening displacement, $\delta$, and the second term captures the change in $\delta$ due to $P(\vec{\xi})$.

During the crack growth from point 'A' to 'B', the work of separation is

$$
W=\int_{0}^{\Delta c} G_{R}(x) d x
$$

and the decrease in the strain energy is

$$
\Delta U=U_{0}-U_{1}
$$

where $U_{0}$ is the strain energy per unit thickness at the onset of the crack advance, which can be derived from Equation (2) as $c_{0} G_{\mathrm{fa}} / 3$, and $U_{1}$ is the strain energy per unit thickness after the crack front is arrested in a matrix. According to the principle of superposition, $U_{1}$ consists of the contributions from $\delta_{\mathrm{o}}$ and $P(\vec{\xi})$. The former, according to Equation (2), is $c_{1} G_{\mathrm{m}} / 3$, where $c_{1}=c_{0}+\Delta c$, and the latter can be obtained as

$$
\frac{1}{L}\left\{\frac{1}{2} \int_{\Gamma^{\prime}} P(\vec{\xi}) \cdot \delta(\vec{\xi}) d \vec{\xi}\right\}
$$


where, as discussed in Equation (9),

$$
\delta(\vec{\xi})=\frac{2(1-v)}{\mu} \hat{K}(\Delta c) \sqrt{\frac{\xi_{1}+\Delta c}{2 \pi}},
$$

with $\mu$ being the shear modulus [16]. Since the energy dissipation must be balanced by the decrease in the strain energy, $W=\Delta U$, which leads to

$$
\frac{1}{3}\left(c_{0} G_{\mathrm{fa}}-c_{1} G_{\mathrm{m}}\right)=\int_{0}^{\Delta c} G_{\mathrm{R}}(x) d x+\frac{1-v}{L \mu} \hat{K}(\Delta c) \int_{\Gamma^{\prime}} P(\vec{\xi}) \sqrt{\frac{\xi_{1}+\Delta c}{2 \pi}} d \vec{\xi}
$$

With the given values for $\rho$ and $a / L$ ratio, substituting Equations (6) and (12) into (7) and (8) gives $G_{\mathrm{fa}}$ and $\Delta c$.

As shown in Figure 1, the origin of $\vec{x}$ and $\vec{\xi}$ is set on the advancing crack front and can be described as:

$$
\left(\frac{\xi_{1}+x-b}{b}\right)^{2}+\left(\frac{\xi_{2} \pm j L}{a}\right)^{2}=1
$$

with $j=0,1,2, \ldots$ indicating different fiber bundles. For the integration over $\Gamma$, the effect of the fiber bundles more than $5 L$ away is neglected, i.e., $j \leq 5$. In order to solve the equations numerically, Equation (9) is decomposed into a set of algebra equations using the Ritz method

$$
2 \hat{K}(x) \sqrt{\frac{x_{1}^{(i)}}{2 \pi}}+\int_{\Gamma} \hat{H}\left(\vec{x}^{(i)}, \vec{\xi}\right) P(\vec{\xi}) d \vec{\xi}=0
$$

where $\vec{x}^{(i)}(i=1,2, \ldots, n)$ are the $n$th points in the right-hand side of $\Gamma^{\prime}$. The form of $P(\vec{\xi})$ is taken as a $m$ th order polynomial of $\vec{\xi}$. The coefficients are determined through the accelerated relaxation algorithm [17]. The values of $n$ and $m$ are set to 10 and 4, respectively, a further increase of which only causes negligible variations in $\Delta c$ and $G_{\mathrm{fa}}$.

\section{INFLUENCE OF FIBER BUNDLE GEOMETRY ON FRACTURE RESISTANCE}

Figure 3 shows the numerical result of $G_{\mathrm{fa}}$ as a function of $D$ and $\rho$, with $D=a / L$ being the size/spacing ratio of the fiber bundles. If the fiber bundles could be cut through by the crack front, the fracture resistance, $G_{\mathrm{fa} 0}$, is no longer determined by the trapping effect. Instead, there exists an analytical solution accounting for the additional work of separation associated with the fiber breakage [9]

$$
\frac{G_{\mathrm{fa} 0}}{G_{\mathrm{m}}}=(1-2 D)+\left(\frac{K_{\mathrm{IC}}^{\mathrm{F}}}{K_{\mathrm{IC}}^{\mathrm{m}}}\right)^{2}(2 D)
$$




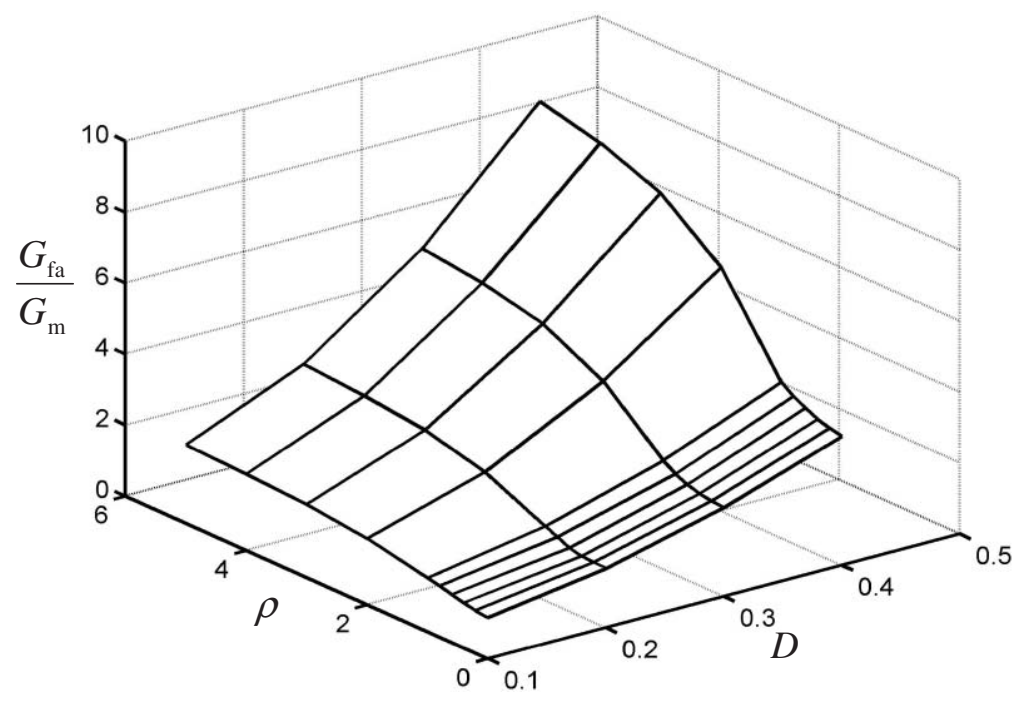

Figure 3. The effects of the size/spacing ratio, $D$, and the cross-sectional aspect ratio, $\rho$, of fiber bundles on the crack trapping effect.

where $K_{\mathrm{IC}}^{\mathrm{F}}$ is the fracture toughness of the fiber and $K_{\mathrm{IC}}^{\mathrm{m}}$ is the toughness of the matrix. When $K_{\mathrm{IC}}^{\mathrm{F}}$ is relatively small, $G_{\mathrm{fa} 0}$ increases with $K_{\mathrm{IC}}^{\mathrm{F}}$; when $K_{\mathrm{IC}}^{\mathrm{F}} \geq K_{\mathrm{IC} 0}^{\mathrm{F}}, G_{\mathrm{fa} 0}$ becomes independent of $K_{\mathrm{IC}}^{\mathrm{F}}$, with $K_{\mathrm{IC} 0}^{\mathrm{F}}$ being the critical fiber toughness above which the fibers become impenetrable. In the latter case, the toughening effect is dominated by the crack trapping effect. Thus, $G_{\mathrm{fa}}$ can be expressed as [6]:

$$
\frac{G_{\mathrm{fa}}}{G_{\mathrm{m}}}=(1-2 D)+\left(\frac{K_{\mathrm{IC} 0}^{\mathrm{F}}}{K_{\mathrm{IC}}^{\mathrm{m}}}\right)^{2}(2 D)
$$

The ratio of $K_{\mathrm{IC} 0}^{\mathrm{F}} / K_{\mathrm{IC}}^{\mathrm{m}}$ is a function of both $D$ and $\rho$, and can be stated as $[1.0+9.6 D+$ $\left.1.8 D^{2}\right] \cdot f(\rho)$, with the term in the brackets being the critical toughness for circular fibers. By expressing the numerical result of $f(\rho)$ as a power-law type function, we have

$$
\frac{G_{\mathrm{fa}}}{G_{\mathrm{m}}}=(1-2 D)+\left[\left(1.0+9.6 D+1.8 D^{2}\right) \cdot f(\rho)\right]^{2}(2 D)
$$

where

$$
f(\rho)= \begin{cases}\rho^{0.23} & (\rho \geq 1) \\ 1-0.15 \cdot(1-\rho)^{0.30} & (0 \leq \rho<1)\end{cases}
$$

After the trapping effect of an array of fiber bundles in an FRC has been overcome, the fiber bundles act as bridging reinforcements. Eventually when more and more fibers are exposed to the crack plane the fiber breakage will occur, and at the steady state the associated fracture work is $\chi_{0} N(\pi a b)$, where $\chi_{0}$ is the specific work of separation of fibers, and $N$ and $\pi a b$ are the number density and the cross-sectional area of fiber 
bundles, respectively. Consequently, the overall fracture resistance is

$$
\frac{G_{\mathrm{cr}}}{G_{\mathrm{m}}}=(1-2 D)+\left[\left(1.0+9.6 D+1.8 D^{2}\right) \cdot f(\rho)\right]^{2}(2 D)+\chi N(\pi a b)
$$

with $\chi=\chi_{0} / G_{\mathrm{m}}$. In continuous fiber reinforced composites, the fiber volume fraction, $c$, equals the area fraction of the fibers in the fracture surface. Under this condition, Equations (17) and (18) can be rewritten as:

$$
\frac{G_{\mathrm{fa}}}{G_{\mathrm{m}}}=\left(1-\frac{2 c}{\pi D_{b}}\right)+\left[\left(1.0+6.11 \frac{c}{D_{b}}+2.29 \frac{c^{2}}{D_{b}^{2}}\right) \cdot f(\rho)\right]^{2}\left(\frac{2 c}{\pi D_{b}}\right)
$$

and

$$
\frac{G_{\mathrm{cr}}}{G_{\mathrm{m}}}=\left(1-\frac{2 c}{\pi D_{b}}\right)+\left[\left(1.0+6.11 \frac{c}{D_{b}}+2.29 \frac{c^{2}}{D_{b}^{2}}\right) \cdot f(\rho)\right]^{2}\left(\frac{2 c}{\pi D_{b}}\right)+\chi c
$$

where $D_{b}=b / L_{b}$ and $L_{\mathrm{b}}$ is the fiber-fiber distance along the crack propagation direction. Figure 4 shows the relationship between $G_{\mathrm{fa}}$ and $c$.

\section{DISCUSSION}

Figure 3 indicates clearly that $G_{\mathrm{fa}}$ increases with both $D$ and $\rho$. As the size/spacing ratio of the fiber bundles increases, more crack front is directly trapped and therefore a larger 'driving force' is required to overcome the barrier effect. If $D$ is constant and $\rho$ is small, the critical penetration depth for the crack front to bypass the obstacles is low. As $\rho$ becomes larger, the penetrating front must propagate across the 'channels' between the adjacent fiber bundles before the separated sections can merge into a new front. As a result, the degree of nonuniformity of stress intensity distribution along the front is significantly higher and the associated critical energy release rate increases with $\rho$. When $\rho$ is close to 1 , i.e., the cross section is nearly circular, $G_{\mathrm{fa}}$ is most sensitive to $\rho$. When $\rho$ is reduced to close to 0 or increases to much larger than 1 , the sensitivity is lowered considerably. In Tables 1 and 2, the numerical results are compared with the experimental data for reinforcements of $\rho \approx 1$ and $\rho \approx 0$, respectively. The experimental data in Table 1 were obtained in a crack trapping measurement of brittle matrix composites reinforced by circular, tough rods [6], and those in Table 2 are the fracture resistance of persistent grain boundary islands [18]. It can be seen that the theoretical and the experimental results fit with each other quite well.

Figure 4 shows that, as long as the fiber-matrix interface is strong, increasing the fiber volume fraction is always of a beneficial effect to the fracture resistance, as it should. With the same fiber volume fraction, the crack front-fiber interaction can be quite different as $\rho$ varies. With the increase of $\rho$, on the one hand, the size/spacing ratio of the fiber bundles is reduced and the fracture resistance tends to decrease. On the other hand, under this condition the critical penetration depth rises, which tends to cause an increase in $G_{\mathrm{fa}}$. The simulation indicates that $G_{\mathrm{fa}}$ increases with $\rho$, i.e. the dominant 

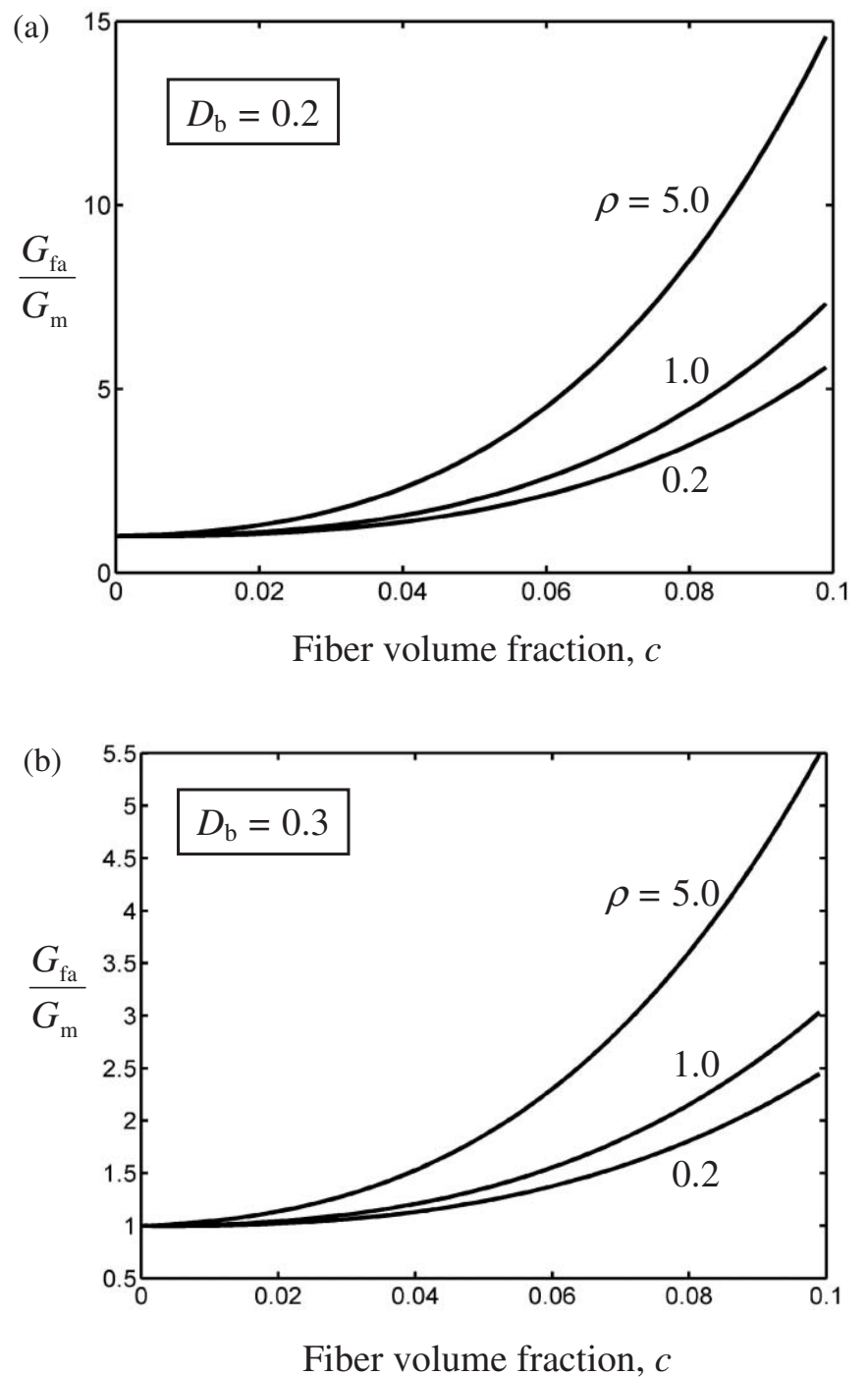

Figure 4. The fracture resistance associated with crack trapping, $G_{f a}$, as a function of the fiber volume fraction: (a) $D_{b}=0.2$; and (b) $D_{b}=0.3$.

mechanism is the latter one. Through the comparison of Figures 4(a) and (b), it can be seen that when $c$ and $\rho$ are constant, decreasing $D_{b}$ is beneficial to the fracture toughness, since it leads to an increase in $D$.

In an isotropic and homogeneous matrix $\mu=E / 2(1+v)$, and therefore, the only elastic parameter present in governing Equations (6)-(8) and (12) is the Poisson's ratio, $v$. According to the numerical results, the change in $G_{\mathrm{fa}}$ is less than $2 \%$ as $v$ varies in the range of 0.01 to 0.49 . Thus, it can be stated that the crack trapping effect of the fiber bundles is independent of the elastic properties of the matrix.

Although in the earlier discussion the variation of the strain energy associated with the crack propagation is analyzed for the DCB sample depicted in Figure 2, 
Table 1. Comparison of the experimental data and the numerical results for the crack trapping effect of reinforcements with $\rho \approx 1$.

\begin{tabular}{llllll}
\hline Size/spacing ratio, $D$ & 0.12 & 0.12 & 0.14 & 0.18 & 0.25 \\
Experimental data of $G_{\mathrm{fa}} / G_{\mathrm{m}}[6]$ & 2.25 & 2.96 & 3.84 & 4.02 & 7.29 \\
Numerical results of $G_{\mathrm{fa}} / G_{\mathrm{m}}$ & 2.38 & 2.38 & 2.61 & 3.91 & 6.87 \\
\hline
\end{tabular}

Table 2. Comparison of the experimental data and the numerical results for the crack trapping effect of reinforcements with $\rho \approx 0$.

\begin{tabular}{lllllll}
\hline Size/spacing ratio, $D$ & 0.061 & 0.071 & 0.074 & 0.080 & 0.105 & 0.126 \\
Experimental data of $G_{\mathrm{fa}} / G_{\mathrm{m}}[18]$ & 1.15 & 1.18 & 1.18 & 1.21 & 1.28 & 1.38 \\
Numerical results of $G_{\mathrm{fa}} / G_{\mathrm{m}}$ & 1.14 & 1.17 & 1.19 & 1.20 & 1.28 & 1.40 \\
\hline
\end{tabular}

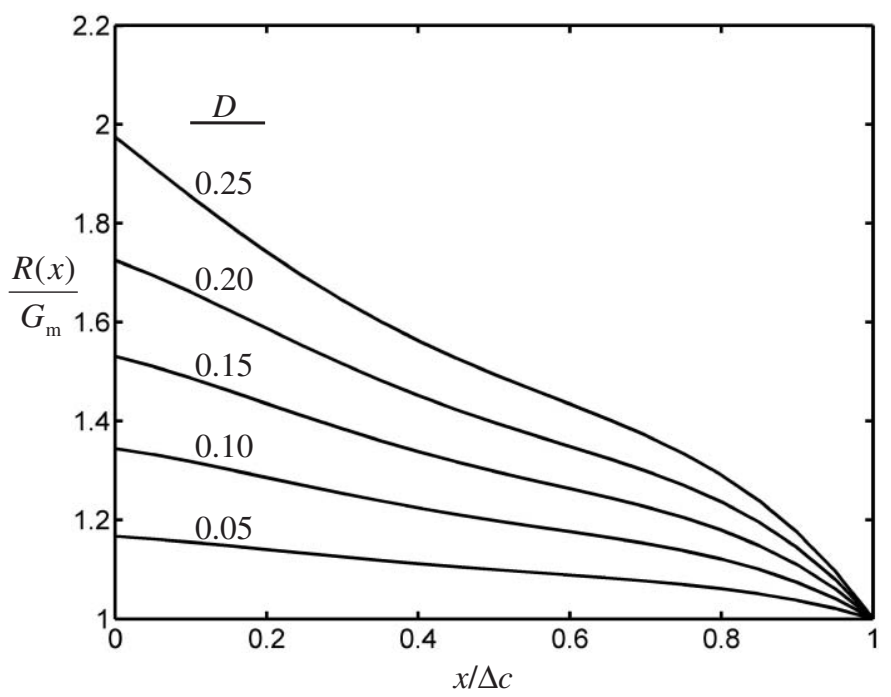

Figure 5. The fracture resistance gradient required to keep the crack advance quasi-static $(\rho=1)$.

most of the geometry factors vanish in the governing equations. Hence, the results of Equations (17)-(20) reflect the material properties. The factor of the initial crack length, however, does come in by affecting $\partial U / \partial c$. In a composite material containing a longer crack, as the crack advances, the rate of strain energy change is lower, which in turn reduces the fracture resistance. This phenomenon can also be attributed to the fact that, at the tip of a longer crack, the fiber bundles 'look smaller'. Nevertheless, the variation in $G_{\mathrm{fa}}$ at different $c_{0}$ in the range of $10 a$ to $1000 a$ is below $5 \%$, that is, the size effect is negligible. Note that if $c_{0}<10 a$, the boundary condition must be taken into account and the model of the infinitely long fiber bundle array depicted is no longer valid.

Figure 5 shows the 'imaginary' local fracture resistance, $R(x)$, between the initial crack tip and the arrested crack tip that is required to keep the crack growth quasi-static. Note that $R(x)$ is always balanced by the crack growth driving force $G_{\mathrm{R}}(x)$, which is determined 
by Equation (6). As the crack propagates, the bridging stress in the fiber bundles is increasingly large while the increase rate keeps decreasing. Since the rate of the background energy release also decreases, the overall energy release rate is lowered with a descending rate, so does $R(x)$.

\section{CONCLUSIONS}

In this article, the cleavage crack propagation across a field of continuous fiber bundles in a brittle matrix composite is analyzed using an energy method. The total fracture work consists of the component of crack trapping and the work of separation associated with the fiber breakage. The factors that govern the toughness include the fiber volume fraction, the cross-sectional aspect ratio of the fiber bundles, and the specific work of separation. The numerical results indicate that with the perfect fiber-matrix interface, the critical energy release rate increases monotonically with the size/spacing ratio of the fiber bundles, and the following conclusions are drawn:

1. With a constant size/spacing ratio, the fracture resistance caused by the crack trapping effect increases with the cross-sectional aspect ratio of the fiber bundles, $\rho$, and is most sensitive to $\rho$ when the cross section is nearly circular.

2. With a constant fiber volume fraction, increasing $\rho$ results in a smaller size/spacing ratio, which tends to lower the fracture resistance. On the other hand, it also leads to a larger critical penetration depth, which is beneficial to the toughening effect. According to the numerical result, the latter mechanism is more important.

3. With a constant fiber volume fraction, an anisotropic fiber bundle distribution, particularly a low number density along the crack propagation direction and a high number density along the cleavage front, can increase the fracture resistance considerably.

4. The crack trapping effect is independent of the crack length and the elastic properties of the matrix.

\section{ACKNOWLEDGMENTS}

This work was supported by the NSF under Grant CMS-0408276.

\section{REFERENCES}

1. Simacek, P. and Karbhari, V.M. (1996). Notes on the Modeling of Preform Compaction. 1. Micromechanics at the Fiber Bundle Level, Journal of Reinforced Plastics and Composites, 15: 86-122.

2. McCartney, L.N. (2003). Physically Based Damage Models for Laminated Composites, PIME LJournal of Materials Design and Applications, 217: 163-199.

3. Kim, J.K. and Mai, Y.W. (1991). High-strength, High Fracture-toughness Fiber Composites with Interface Control - A Review, Composites Science and Technology, 41: 333-378.

4. Cantwell, W.J. and Morton, J. (1991). The Impact Resistance of Composite Materials A Review, Composites, 22: 347-362. 
5. Mouritz, A.P., Baini, C. and Herszberg, I. (1999). Mode I Interlaminar Fracture Toughness Properties of Advanced Textile Fiberglass Composites, Composites Part A, 30: 859-870.

6. Mower, T.M. and Argon, A.S. (1995). Experimental Investigations of Crack Trapping in Brittle Heterogeneous Solids, Mechanics of Materials, 19: 343-364.

7. DiFrancia, C., Ward, T.C. and Claus, R.O. (1996). The Single-fiber Pull-out Test. 1. Review and Interpretation, Composites Part A, 27: 597-612.

8. Nakai, A., Ohki, T., Takeda, N. and Namada, H. (2001). Mechanical Properties and Microfracture Behaviors of Flat Braided Composites with a Circular Hole, Composite Structures, 52: 315-322.

9. Rose, L.R.F. (1987). Toughening due to Crack-front Interaction with a Second-phase Dispersion, Mechanics of Materials, 6: 11-15.

10. Gao, H. and Rice J.R. (1989). A First-order Perturbation Analysis of Crack Trapping by Arrays of Obstacles, Journal of Applied Mechanics, 56: 828-836.

11. Qiao, Y. and Kong, X. (2004). Unstable Crack Advance across a Regular Array of Short Fibers in Brittle Matrix, Composite Science and Technology, 64: 711-717.

12. Qiao, Y., Kong, X. and Pan, E. (2004). Fracture Toughness of Thermoset Composites Reinforced by Strongly Bonded Impenetrable Short Fibers, Engineering Fracture Mechanics, 71: 2621-2633.

13. Lee, D.G., Kim, P.J. and Choi, J.K. (2000). Temperature Rise and Surface Roughness of Carbon Fiber Epoxy Composites During Cut-off Grinding, Journal of Composite Materials, 34: 2061-2080.

14. Headifen, G.R. and Fahrenthold, E.P. (1991). Mechanical and Electrical Properties of Glass and Carbon Fiber Reinforced Composites, Journal of Energy Resources Technology, 113: 176-181.

15. Mizumachi, H. (1997). Adhesion Science and Technology, T\&F STM, Berlin, Germany.

16. Ulfyand, Y.S. (1965). Survey of Articles on the Application of Integral Transforms in Theory of Elasticity, University of North Carolina, Raleigh, NC.

17. Dennis, J.E. and Schnabel, R.B. (1996). Numerical Methods for Unconstrained Optimization and Nonlinear Equations, SIAM, Philadelphia, PA.

18. Qiao, Y. and Argon, A.S. (2003). Cleavage Cracking Resistance of High Angle Grain Boundaries in Fe-3\% Si Alloy, Mechanics of Materials, 35: 313-331. 